\title{
Bridging Phases at the Morphotropic Boundaries of Lead-Oxide Solid Solutions
}

\author{
B. Noheda ${ }^{1}$ and D.E. Cox $^{2}$ \\ ${ }^{1}$ Materials Science Centre, U. of Groningen, Nijenborgh 4, 9747AG Groningen, The Netherlands \\ 2 Physics Department, Brookhaven National Laboratory, Upton, New York 11973, USA
}

\begin{abstract}
Ceramic solid solutions of $\mathrm{PbZr}_{1-x} \mathrm{Ti}_{x} \mathrm{O}_{3}$ (PZT) with compositions $x \simeq 0.50$ are well-known for their extraordinarily large piezoelectric responses. The latter are highly anisotropic, and it was recently shown that, for the rhombohedral compositions $(x \lesssim 0.5)$, the piezoelectric coefficients were largest away from the polar direction, contrary to common belief. Shortly afterwards a low-symmetry monoclinic phase was observed by synchrotron x-ray diffraction at around $x=0.50$. Similar behavior and features are also present in a number of related lead-based strongly-piezoelectric single crystals, such as $\mathrm{Pb}\left(\mathrm{Mg}_{1 / 3} \mathrm{Nb}_{2 / 3}\right)_{1-x} \mathrm{Ti}_{x} \mathrm{O}_{3}, \mathrm{~Pb}\left(\mathrm{Zn}_{1 / 3} \mathrm{Nb}_{2 / 3}\right)_{1-x} \mathrm{Ti}_{x} \mathrm{O}_{3}$, and $\mathrm{Pb}\left(\mathrm{Sn}_{1 / 2} \mathrm{Nb}_{1 / 2}\right)_{1-x} \mathrm{Ti}_{x} \mathrm{O}_{3}$, with piezoelectric coeficients of about $2500 \mathrm{pm} / \mathrm{V}$, the highest values recorded to date. Recent experimental and theoretical work has greatly improved our understanding of these technologically-important systems, but there are still some open questions. In this review we try to summarize the most recent progress in the field.
\end{abstract}

PACS numbers:

\section{INTRODUCTION}

Gen Shirane will be long remembered as one of the pioneers in the application of neutron scattering techniques to phase transitions. It may not be so widely appreciated that he was also a pioneer in the first studies on the highly piezoelectric ceramics of $\mathrm{PbZr}_{1-x} \mathrm{Ti}_{x} \mathrm{O}_{3}$ (PZT), which he and his colleagues carried out in Japan in the early 1950's [1-4]. When he once more took up the study of piezoelectric systems in the last few years of his life, he was again one of the pioneers in the renaissance of this field.

Over the many years that passed since his earlier work, it came to be generally accepted that the so-called morphotropic phase boundary (MPB) in PZT at $x \approx 0.50$, which is associated with a number of remarkable dielectric and piezoelectric properties, is a region of coexistence between the tetragonal, Ti- rich $(\mathrm{T})$ and rhombohedral, Zr-rich $(\mathrm{R})$ phases [5] with a variable width dependent on the homogeneity of the powders [6-8] and the grain size[9]. It should be noted that no single crystals of PZT in the morphotropic region are available. A comprehensive review of the early research on the MPB can be found in a recent paper by Glazer et al. [10].

In the late 1990's a renewed interest in piezoelectricity was awakened due mainly to work from the MRL at Pennsylvania State University. Park and Shrout [11] reported on high-strain piezoelectric rhombohedral single-crystals of $\mathrm{Pb}\left(\mathrm{Mg}_{1 / 3} \mathrm{Nb}_{2 / 3}\right)_{1-x} \mathrm{Ti}_{x} \mathrm{O}_{3}(\mathrm{PMN}-\mathrm{xPT})$ and $\mathrm{Pb}\left(\mathrm{Zn}_{1 / 3} \mathrm{Nb}_{2 / 3}\right)_{1-x} \mathrm{Ti}_{x} \mathrm{O}_{3}$ (PZN-xPT) with electromechanical strain values of up to $0.6 \%$, little hysteresis, and piezoelectric coefficients of about $2500 \mathrm{pm} / \mathrm{V}$ when oriented along the [001] direction. These crystals can also show huge electromechanical strains of up to $1.7 \%$ under an [001] electric field related to the induced R-T transformation [12]. Park and Shrout explained the lack of hysteresis as being due to a domain engineered state in which the rhombohedral domains are symmetrically oriented to produce a macroscopic tetragonal symmetry. Within each domain the polarization would rotate under the [001] field to align closer to the field direction. At about the same time Du et al. showed, using a phenomenological approach, that rhombohedral PZT also exhibits maximum piezoelectric response under an electric field oriented along the [001] direction, and not along the polar [111] direction [13], which was later confirmed experimentally [14-16].

Shortly afterwards, a hitherto unsuspected monoclinic (M) phase was observed at the MPB by high-resolution synchrotron x-ray powder diffraction techniques [17]. The symmetry of the new phase was found to be very low space group $\mathrm{Cm}$, which has just a mirror plane and no symmetry axis. This mirror plane is also the only symmetry element common to the well-known $\mathrm{R}$ (space group $R 3 \mathrm{~m}$ ) and $\mathrm{T}$ (space group $P 4 \mathrm{~mm}$ ) phases, so the M phase can be viewed as a bridge between these two structures. In the powder diffractograms the monoclinic phase has some very distinctive features. Although from a casual inspection of just the pseudocubic (hh0) and (hhh) reflection profiles it might be possible to mistakenly infer the coexistence of tetragonal and rhombohedral phases, a careful consideration of the full pattern makes it clear that this cannot be the case. In particular, the well-defined doublets at the pseudocubic (h00) reflections (see, e.g., Fig. 2 in Ref. [18]) demonstrate that the R-T coexistence model has to be discarded. The lattice parameters and structure of the $x=0.48$ monoclinic phase in PZT were determined at $20 \mathrm{~K}$ from a Rietveld 


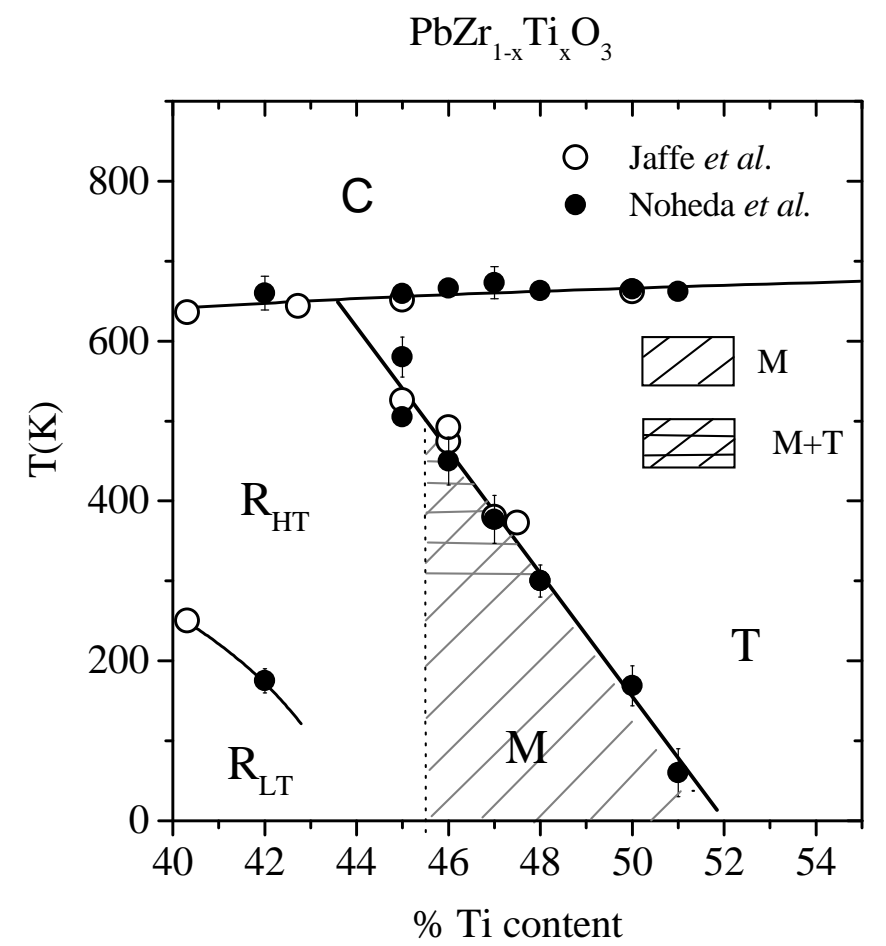

FIG. 1: Phase diagram of $\mathrm{PbZr}_{1-x} \mathrm{Ti}_{x} \mathrm{O}_{3}$ around the MPB, as proposed in Ref. [19] based on synchrotron x-ray powder diffraction data.

profile analysis of the whole x-ray diffractogram [19]. The full diffraction pattern was well explained by an $\mathrm{M}(\mathrm{Cm})$ phase in which the polar axis is tilted $24^{\circ}$ away from the $[001]_{p c}$ direction towards the $[111]_{p c}$ direction, showing the bridging role of this intermediate phase. In subsequent work, the lattice parameters were measured as a function of temperature and composition, and a phase diagram around the morphotropic region was proposed, as shown in Fig. 1 [18]. However, recent neutron studies have shown the presence of a few very weak superlattice peaks which are not accounted for by this simple model, as will be discussed in detail in the next Section.

Around the same time, $\mathrm{Fu}$ and Cohen [20], using first-principles calculations on $\mathrm{BaTiO}_{3}$, reported that the high electromechanical response observed in rhombohedral crystals under an electric field oriented along the [001] axis was due to the rotation of the polarization within the plane defined by [001] and [111]. This is precisely the monoclinic plane of PZT! Thus the intermediate states induced by a field would have monoclinic symmetry. This led to the postulation $[15,21]$ that the presence of a monoclinic phase at the MPB was the direct cause of the great enhancement of the piezoelectric response long known in this compositional region.

First-principles calculations by Bellaiche et al. [21] reproduced the stability of the monoclinic phase in a narrow region at the MPB when a random $\mathrm{Zr} / \mathrm{Ti}$ cation distribution was taken into account. The stability of a monoclinic phase of space group $C m$ (the so-called $\mathrm{M}_{A}$ phase) in between the $\mathrm{R}$ and $\mathrm{T}$ phases was also explained using a LandauDevonshire approach by Vanderbilt and Cohen[22], who furthermore predicted two other monoclinic phases $\left(\mathrm{M}_{B}\right.$ and $\mathrm{M}_{C}$ ) bridging the rhombohedral and orthorhombic, and the orthorhombic and tetragonal phase of perovskites, respectively. These two latter monoclinic phases have since been observed in PMN-xPT and PZN-xPT[23-35] and, more recently in $\mathrm{Pb}\left(\mathrm{Sc}_{1 / 2} \mathrm{Nb}_{1 / 2} \mathrm{O}_{3}\right)_{1-x} \mathrm{Ti}_{x} \mathrm{O}_{3}$ (PSN-PT) [36, 37].

An earlier review can be found in Ref. [38], but since then numerous papers have been published on the topic and new ideas have been advanced. Due to the intrinsic cation disorder in these lead oxide solid solutions, the average structure differs from that at the level of the unit cell. Small domains and strain effects can result in a markedly different picture also at the mesoscopic scale. In general, one can say that current work on the topic is directed towards the observation of the MPB regions by different techniques and thereby obtain a consistent picture of the phenomena observed at the different length scales. Furthermore, there exist also concerns about the stability of the intermediate 
monoclinic phases[39, 40], as well as about whether the high electromechanical response of these compounds is mainly intrinsic or extrinsic. These issues are still not fully resolved but there has been substantial progress in the field. In the following sections we will try to give a general idea of the most recent developments.

\section{STRUCTURAL INSTABILITIES AT THE MPB}

\section{A. Oxygen Octahedral Tilts}

First-principles calculations by Fornari and Singh [41] have shown that, in ferroelectric perovskites, strain can induce local anti-ferrodistortive tilts of the oxygen octahedra in coexistence with the ferroelectric cation displacements. Such tilts would be expected to lower the symmetry of the lattice, and indeed evidence of a cell-doubling transition in PZT with $x=0.48$ at low temperatures was reported by Ragini et al. [42] from electron diffraction measurements, and by Noheda et al. [18] from neutron powder diffraction data even though no indication of such a transition was observed in the corresponding x-ray powder patterns due to the low scattering power of oxygen.

However, detailed Rietveld analysis of the neutron powder data from the $x=0.48$ composition has proved to be far from straightforward. Although the reported raw data appear to be very similar at first sight, there are currently three different interpretations. The neutron patterns are characterized by the appearance of one or more weak superlattice peaks, indicating the doubling of the monoclinic unit cell along the $c$-axis, as reported in Ref. [18], and by Ranjan and coworkers $[43,44]$, who concluded the low-temperature structure was also monoclinic, space group $C c$, with a doubled cell characteristic of antiphase tilting of the oxygen octahedra. On the other hand, Frantti et al. [45] have concluded that the weak superlattice peaks should be attributed to a minority rhombohedral phase with $R 3 c$ symmetry in coexistence with the monoclinic $\mathrm{Cm}$ phase. However, this conclusion was not supported by electron diffraction data obtained by Noheda et al. [46], which showed no evidence of a rhombohedral phase, but instead the $C m$ phase in coexistence with a minority $C c$ phase. Furthermore, a similar coexistence model was deduced from a recent Rietveld analysis of the neutron data mentioned in Ref. [18], namely monoclinic $C m$ and $C c$ phases in the approximate ratio 4:1 [47]. At about the same time, Ranjan et al. [48] reported that the previously published neutron data [43, 44] also favored a coexistence model, but with a markedly different ratio of $C m$ and $C c$ phases, in this case about $1: 2$. In both studies it was concluded that a minority $R 3 c$ phase fails to account for the superlattice peak positions, but this is disputed by Frantti et al. [49], who argue that if anisotropic peak broadening is allowed for correctly [50], a coexistence model of $C m$ and $R 3 c$ phases accounts well for the peak positions and intensities. [51] In a very recent paper, published during the writing of this manuscript, Woodward et al.[52] have presented a detailed analysis of TEM measurements across the phase diagram of PZT and propose a single Cc phase around the MPB at sufficiently low temperatures. These authors point out that the monoclinic Cc phase supports the tilt system $a^{-} b^{-} b^{-}$(in Glazer's notation[53]), which is more general than those previously considered. If so, the Cc phase can then be considered as the structural bridge between the R3c $\left(a^{-} a^{-} a^{-}\right.$tilted $)$and the untilted Cm phase.[52]

Thus it must be said that the current situation is rather confusing, and illustrates the difficulty of obtaining a definitive result from Rietveld analysis alone in these complicated piezoelectric systems, which are typically characterized by low symmetry, local order, pronounced microstructural effects, and coexistence of two (or more) closely-related phases. Nevertheless, we believe that although oxygen tilts may play an important role in lowering the elastic energy of the system, and in stabilizing the monoclinic phase[41], it is the symmetry element of the cation displacements (i.e. the mirror plane of the simple monoclinic phase, $\mathrm{Cm}$ ) which defines the symmetry of the polarization and the anisotropy of the physical properties.

Further insight into the structural relationships in the PZT system has been obtained from diffraction experiments carried out under hydrostatic pressure. From synchrotron x-ray powder data and Raman spectroscopy studies, aided by first-principles calculations, of a sample with $x=0.48$ at room temperature, Sani et al.[54] have shown that polarization rotation from the $\mathrm{T}$ towards the $\mathrm{R}$ phase, through the monoclinic $\mathrm{M}_{A}$ phase, occurs under pressure, together with tilting of the oxygen octahedra in agreement with Ref. [41]. Neutron and x-ray measurements on a series of PZT compositions by Rouquette and colleagues [55] have confirmed both the rotation within the $\mathrm{M}_{A}$ phase and the oxygen tilts; in particular, for $x=0.60$ there is a transition from $\mathrm{T}$ to $\mathrm{M}_{A}$ symmetry around 1 GPa, followed by a transition to the antiferrodistortive $C c$ structure at about $3 \mathrm{GPa}$. In a subsequent neutron study of an $x=0.48$ sample [56], a series of monoclinic $(C m$ and $C c)$ and triclinic $(F 1$ and $F \overline{1})$ phases were reported, and the data suggest that a rhombohedral phase is never reached. Recently $\mathrm{Wu}$ and Cohen have reported that a T-M-R-C sequence can be induced by pressure in pure $\mathrm{PbTiO}_{3}$. Thus hydrostatic and chemical (Zr-substitution) pressure have a similar effect on $\mathrm{PbTiO}_{3}$, and a MPB in between the $\mathrm{T}$ and $\mathrm{R}$ phases is predicted at about 12 GPa at low temperatures [57]. 


\section{B. Electric Field Effects on the MPB}

To investigate the effect of an electric field on the behavior of the MPB, x-ray diffraction patterns from ceramics of rhombohedral and tetragonal PZT were measured before, during and after poling. The effect of the poling was found to be irreversible and consistent with the induction of an $\mathrm{M}_{A}$ type distortion[15]. No elongation of the unit cell along the polar directions was observed. The piezoelectric coefficient measured from the patterns of the rhombohedral composition along [001] was $500 \mathrm{pm} / \mathrm{V}$, surprisingly, about equal to that obtained by dilatometry. The availability of single crystals of PMN-xPT and PZN-xPT, has allowed the induced symmetry changes to be followed directly in-situ under an E-field, and showed that close to the MPB the initial R phase was indeed irreversibly transformed into $\mathrm{M}\left(\mathrm{M}_{A}\right.$ or $\left.\mathrm{M}_{C}\right)$ by applying an [001] E-field, and that the monoclinic phases acted as bridging phases in the rhombohedal-tetragonal transformation not only as a function of composition but also under an applied electric field [31, 58-60], in excellent agreement with theoretical calculations[61]. Viehland and co-workers showed that this behaviour was not specific to the [001] oriented crystal, and that a [101] E-field could induce the rhombohedralorthorhombic transformation and giant piezoelectric deformations as well[62, 63]. In particular, Cao et al.[63] have shown that electric field oriented in this direction can induce the rarely-observed monoclinic $\mathrm{M}_{B}$ phase, predicted by Vanderbilt and Cohen[22]

\section{Stability Issues at the MPB}

As mentioned above, theoretical calculations fully support the stability of the M phase near the MPB under zero field and further away from the MPB under a properly oriented electric field. Renault et al. have checked the fieldinduced monoclinic phase in PZN-9PT monodomain crystals after removal of the field, finding that the monoclinic phase is stable and the samples do not show partial depoling [64]. Topolov has interpreted the primary role played by the monoclinic phase as elastic matching and strain relief during the R-T transformation [65, 66], which has found direct experimental support from Frantti et al.[67]. However, there is in the experimental literature some divergence concerning the stability of the $\mathrm{M}$ phase. Until now, an indication of the monoclinic phase in PZT has been reported by several techniques[68-70], but it is fair to say that the evidence is somewhat elusive. Only high-resolution synchrotron or neutron diffraction measurements at low temperatures on high quality powders are able to show the $\mathrm{M}$ phase definitively due to the small distortion with respect to the tetragonal unit cell. Coexistence with the $\mathrm{R}$ and $\mathrm{T}$ phases has often been reported, but it is primarily in very homogeneous samples with compositions around $x=0.48$ at low temperatures where the $\mathrm{M}$ phases $(C m / C c)$ show up most distinctly. X-ray diffraction from laboratory sources has most often not been capable of directly resolving the subtle $\mathrm{M}$ splittings, and the best evidence has been provided by the improved fits obtained from Rietveld analysis of the whole profile when the M phase is considered[42, 71]. Moreover, the stress dependence of the $\mathrm{M}$ phase discussed above explains why in certain ceramics with relatively small grain size and relatively large microstrain, the low temperature monoclinic phase was not present and, instead, a rhombohedral phase was observed[72].

As will be discussed later, it is worth noting that, due to the near-degeneracy of the different phases at the MPB, small differences in the strain state of the samples can easily alter the phase stability and thus explain the reported divergence in the experimental results. Accordingly, special attention must be given to sample preparation. In principle, the structure of PMN-xPT or PZN-xPT single crystals can be determined by crushing a small piece of the crystal to a fine powder, but to obtain a suitably orientationally-averaged powder sample is likely to result in peak broadening because of the induced strain. Although the M phases might still be present[73], such broadening can make it very hard to resolve the small monoclinic splittings in the diffraction pattern. One compromise which was found to yield extremely narrow and well-resolved peaks, and hence to allow an accurate unit cell determination involves careful crushing and sieving of an $\sim 40 \mu \mathrm{m}$ fraction[25, 73]. However, there is the risk with this technique that the powder averaging may not be sufficiently good to permit a reliable analysis of the atomic positions. Thus it now appears to be widely accepted that, given the delicate energy balance at the MPB and the large microstrains involved, the microstructure of the materials will be a decisive factor in the phase stability of the different phases around the MPB, explaining the relatively diverse results in the literature, including the dependence with grain size of the tetragonal-monoclinic ratio [74], and the monoclinic-rhombohedral transformation induced by grinding[73]. Moreover, it has been shown that in single crystals of PZN-PT and PMN-PT the structure of the outermost region of the crystal is different from that of the inner part[75-77, 79]. This 'skin effect'is responsible for some striking differences observed between neutron and x-ray (at both medium and low energies) diffraction data due to the very different penetration depths $[58,73,80]$, and could also be the cause of the differences encountered between single- 
a)

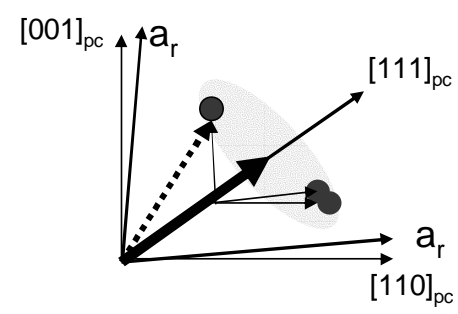

b)

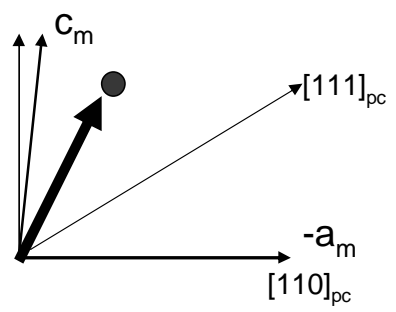

c)

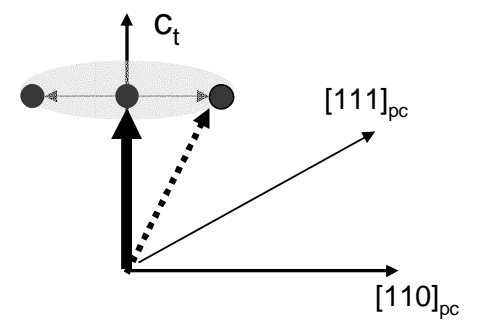

FIG. 2: Monoclinic plane of $\mathrm{PbZr}_{1-x} \mathrm{Ti}_{x} \mathrm{O}_{3}$, containing the rhombohedral and tetragonal polar axes. Pb shifts from the highsymmetry positions are sketched as inferred from Rietveld analysis of synchrotron and neutron diffraction powder diffraction data, showing: (a) The three equivalent sites proposed by Corker et al. [85] to explain the disorder in the rhombohedral phase (b) $\mathrm{Pb}$ shifts in the monoclinic phase (c) four equivalent sites in the tetragonal phase. Figure adapted from [19].

crystals and ceramics. An extensive review of the skin effect in piezoelectrics crystals is presented by Xu et al. in this issue.

\section{LOCAL VERSUS AVERAGE SYMMETRY LOWERING}

Theoretical investigations point to the importance of cation disorder in explaining the features of the MPB[21, 81]. Because of the large coherence length of neutrons and x-rays, diffraction data from these probes are only capable of determining average distortions. However, because information about static and dynamic disorder in the average structure, or short-range order (shorter than the coherence length of the probe), is contained in the so-called anisotropic displacement factors, or thermal parameters, useful information can be gained about the local structure by carefully looking at these parameters[82]. That the rhombohedral phase of PZT involved more than just a long-range rhombohedral distortion was first pointed out by Glazer et al. [83], when they observed that the anisotropic displacement factors for the $\mathrm{Pb}$ atoms determined from the neutron diffraction patterns of $\mathrm{PbZr}_{0.9} \mathrm{Ti}_{0.1} \mathrm{O}_{3}$ were unphysically large and highly anisotropic. They also found, in agreement with earlier work by Sawaguchi[4], that they had the shape of ellipsoids flattened perpendicularly to the polar [111] direction. This disorder seemed to be static as it showed little change with temperature. Similar local shifts of $\mathrm{Pb}$ atoms were also found by Teslic et al. using a pair-distribution function analysis [84]. Following up on these findings, in 1998 Corker et al.[85] proposed a model in which the $\mathrm{Pb}$ atoms randomly occupy three different sites shifted, respectively, along pseudocubic [100], [010] and [001] with respect to a common [111] displacement. The random occupancy of these three sites leaves the average shift along the [111] direction unchanged. Thus the average long-range rhombohedral symmetry is maintained, but at the level of the unit cell the symmetry is lowered, as illustrated in Fig. 2.

When the monoclinic phase was observed shortly afterwards in $\mathrm{PbZr}_{0.52} \mathrm{Ti}_{0.48} \mathrm{O}_{3}$ at low temperatures[17, 19], it was found that the monoclinic displacements corresponded very closely to one of the three sites, i.e. [111] $+[001]$, proposed by Corker et al. to explain the local disorder in the rhombohedral phase [85]. The extra [001] shift with respect to an average rhombohedral phase can equally well be viewed as a distortion from tetragonal symmetry by rotation of the polarization away from [001] towards [111], so that the monoclinic phase provides the structural bridge that allows an average rhombohedral structure to change continuously to an average tetragonal structure through the MPB. The values obtained for the anisotropic displacement factors in the M phase did not indicate any substantial differences between local and average order in the monoclinic composition. This was not the case, though, for the tetragonal phase observed at $325 \mathrm{~K}$ in this sample, for which strongly anisotropic displacement factors were observed perpendicular to the polar [001] direction[19].

If the model of Corker et al. is adapted to the tetragonal phase, it is necessary to consider four equivalent sites that, randomly occupied, would allow the average tetragonal structure to be retained. When these sites were chosen to lie along [110], [110], [110] and [110] the Rietveld refinement was significantly improved, and reasonable displacement 
factors were obtained, analogous to the improvement found for the rhombohedral phase [85]. Any one of these four shifts by itself would result in a monoclinic distortion, and thus provide a structural bridge linking the tetragonal to the rhombohedral structure by polarization rotation. This led to the proposal [19] that, as the Ti content is changed across the MPB, one of the local sites of the $\mathrm{R}$ or the $\mathrm{T}$ phase is preferred, resulting in a monoclinic phase in a narrow intermediate region of composition.

Neutron studies by Dmowski et al. using the atomic pair distribution function technique (PDF), which provides details of the distribution of atomic density as a function of interatomic distance without symmetry assumptions, have shown that the local changes in PZT across the MPB are very gradual[86]. In particular, from a comparison of the PDF of PZT with that of pure $\mathrm{PbTiO}_{3}$ and pure $\mathrm{PbZrO}_{3}$, it was suggested that the local environment of the $\mathrm{Zr} / \mathrm{Ti}$ cations remains relatively invariant of composition. They found that $\mathrm{Ti}$ is always ferroelectrically active across the phase diagram, while $\mathrm{Zr}$ is not. The variation with composition is thus due mainly to the $\mathrm{Pb}$ cations, which are locally displaced along the $<100>$ and $<110>$ directions, as in $\mathrm{PbTiO}_{3}$ and $\mathrm{PbZrO}_{3}$, respectively, in order to form four, instead of three, short $\mathrm{Pb}-\mathrm{O}$ bonds (as would result from $<111>$ displacements). The most significant change with $\mathrm{Zr}-\mathrm{Ti}$ content was the distribution of those displacements, i.e., from a majority of $\langle 110\rangle$ displacements close to $x=0$ to a majority of $<100>$ displacements close to $x=1$. There are similarities between the model of Dmowski et al. for the MPB and that discussed earlier, and both propose gradual local changes across the MPB. The main difference between the two is that while the anisotropic displacement factors show an increase in order at the MPB, the PDF studies seem to indicate a larger amount of disorder at the MPB.

By means of x-ray and neutron diffraction measurements on the related solid solution PSN-PT, Haumont et al. have also observed that the R-T transformation as a function of Ti content is gradual[36]. These authors have proposed that for low titanium contents, there is a macroscopic rhombohedral state with local monoclinic symmetry resulting from the combination of $\mathrm{Pb}$ and $\mathrm{Sc} / \mathrm{Nb} / \mathrm{Ti}$ shifts along the [001] and [111] directions, respectively. Since, as in the synchrotron studies of PZT, the disorder is observed to decrease from the rhombohedral side towards the MPB, it is suggested that, with increasing Ti content, competition between cations leads to an increase in the coherence length of the short-range monoclinic phase, which becomes long- range in the morphotropic region.

Recently, Glazer et al. have carried out detailed electron diffraction measurements on PZT across the MPB[10]. The diffuse scattering in these patterns clearly shows the change from short-range order to long-range order as the MPB is approached from both the Zr-rich and the Ti-rich sides. The same authors identify the nature of this short-range order as due to ordered cation displacements rather than compositional inhomogeneity, because of the absence of diffuse scattering along high-symmetry directions. Based on Rietveld profile analysis of x-ray powder data, Ragini et al.[71] have gone further in suggesting that the nominally rhombohedral compositions with $0.47 \geq x \geq 0.38$ are more likely to be 'truly' (long-range as well as short-range ordered) monoclinic. As Glazer et al.[10] have pointed out, a model of uncorrelated monoclinic unit cell distortions throughout the phase diagram with correlation lengths increasing towards the MPB, provides a more plausible explanation of the rather diverse experimental findings concerning the details of the phase stability and coexistence, since these would be very much dependent on the sample homogeneity, and even more on the coherence length of the experimental probes. Overall, this picture can explain the lack of a well-defined R-M phase boundary (indicated by the dotted line in Fig. 1), or any piezoelectric and dielectric peaks for that composition $(\mathrm{x} \simeq 0.455)$.

This model is also attractive in considering the effect of an electric field in these systems. When an [001]-oriented field is applied to the so-called rhombohedral phase, the [001] local shift will be stabilized with respect to the other two, and a long-range monoclinic phase will be induced through polarization rotation[15]. In this case the macroscopic field-induced rhombohedral-to-monoclinic phase transition would actually correspond to a short-range-to-long-range monoclinic phase transformation, and the electric field would have the effect of increasing the correlation length. Guo et al.[15] showed that the piezoelectric elongation of rhombohedral PZT along [111] was undetectable and also that the piezoelectric elongation of tetragonal-monoclinic PZT ( $x=0.48$ at room temperature) was minimal along the [001] direction. These results are consistent with the stabilization of one of the local monoclinic displacements by a properly oriented field on both sides of the MPB.

Using density-functional theory calculations, Grinberg et al.[87] have shown that in PZT (and similarly in PMN-xPT and PZN-xPT[88]) $\mathrm{Pb}<111>$ displacements are indeed avoided, as indicated by the experiments. The effectiveness of the method to deal with related $\mathrm{Pb}$ and $\mathrm{Bi}$ complex oxides is illustrated by its predictive power[89]. The calculations also reveal that the $\mathrm{Pb}$-shifts deviate from the average polar direction, shifting towards the neighbouring $\mathrm{Ti}$ atoms and away from the Zr atoms, due to the increased repulsion with the larger cations (see Figure 3), while the shifts of the $\mathrm{Zr} / \mathrm{Ti}$ atoms do not change significantly with composition. A majority of Ti-rich, Zr-rich or neutral faces 


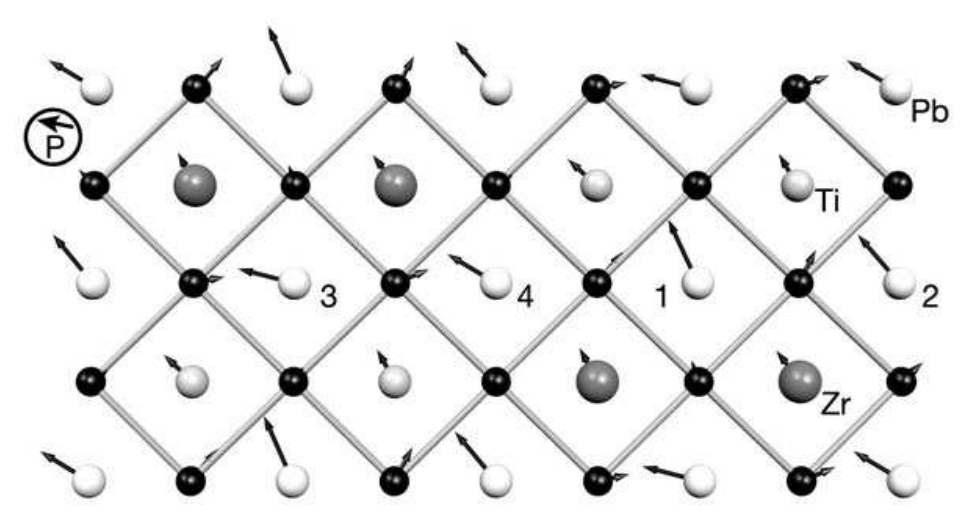

FIG. 3: Local displacements in $\mathrm{PbZr}_{1-x} \mathrm{Ti}_{x} \mathrm{O}_{3}$ resulting from Density Functional Theory calculations free from symmetry constraints by Grinberg et al.[87](reproduced with Nature's and authors' copyright permission)

in the material, towards which the $\mathrm{Pb}$ can shift, will therefore decide whether the average symmetry is tetragonal, rhombohedral or monoclinic, respectively. According to these results, maximum disorder exists in the rhombohedral phase, with a substantial decrease in the monoclinic phase, as experimentally observed. But, in contrast to Refs. [10] and [19], the calculations show minimum disorder in the tetragonal phase.

The latter result is in agreement with the work by Haumont et al.[36] on PSN-PT and that of Ragini et al.[71] on PZT, who have carried out Rietveld analysis of neutron and x-ray diffraction patterns of several compositions across the MPB. In contrast to the previous synchrotron work described above performed on tetragonal PZT with $x=0.48$ at $325 \mathrm{~K}$ [19], at room temperature Haumont et al. and Ragini et al. did not find highly flattened displacement factors in the tetragonal compositions, indicative of little difference between the local and average order on the tetragonal side of the MPB. Molecular-dynamics simulations performed by Shin and collaborators in equilibrated $4 \times 4 \times 4$ supercells of $\mathrm{PbTiO}_{3}$ at $300 \mathrm{~K}$, give relatively large standard deviation values for $\mathrm{Pb}$ displacements $(0.105 \AA, 0.102 \AA, 0.099 \AA)$, corresponding to slight flattening perpendicular to the polar axis, indicating that the origin of the local displacements observed at $325 \mathrm{~K}$ in morphotropic PZT could be partially dynamic[91]. A true phase boundary between the $\mathrm{M}$ and $\mathrm{T}$ phases, where a second-order phase transition is predicted[22], would define the MPB as a critical line, and the only quasi-vertical boundary, in the $x-T$ diagram. This possibility is very attractive, since both elastic softening and the largest anomalies in the piezoelectric and dielectric responses are expected at a second-order phase transition, while still allowing continuous rotation of the polarization.

Continuous rotation of the macroscopic polarization across the MPB due to the rotation of the Ti-shifts in PZT has recently been proposed by Cao et al. [92] based on x-ray absorption fine structure (XAFS) data. These data indicate a continuous change in the Ti local displacements from [001] to [111] for compositions between $x=0.55$ and $x=0.40$. In agreement with Corker et al. [85] and Dmowski et al. [86], these authors find no evidence of substantial ferroelectric shifts of the $\mathrm{Zr}$ atoms. These results thus support the idea that the local Ti dipoles follow the average structural distortion and that the direction of the "local" dipole moments and the macroscopic polarization pretty much coincide.

Another very appealing interpretation of the monoclinic phase has been given by Viehland, Jin and coworkers $[93,94]$. Their model is based upon the assumption that, for such a polarization rotation mechanism, associated with a very small domain wall energy[11], since the different polarization orientations are nearly degenerate, the ferroelectric phase transforms into an inhomogeneous microdomain state which is macroscopically homogeneous, a so-called adaptive phase. This state is described as a miniaturized tetragonal microdomain structure governed by lattice accommodation under stress and electric field. Such an adaptive phase, formed by plates containing twinrelated domains, would be observed as a homogeneous monoclinic phase by diffraction measurements. In order to accommodate the stress and avoid misfits along the domain boundaries, certain relationships between the lattice parameters of the tetragonal phase $\left(a_{t}, c_{t}\right)$ and the adaptive phase $\left(a_{a d}, b_{a d}, c_{a d}\right)$ need to be fulfilled; in particular: $a_{a d}+c_{a d}=a_{t}+c_{t}$ and $b_{a d}=a_{t}$. Moreover, in the $\mathrm{M}_{C}$ phase, $a_{a d}$ and $c_{a d}$ would form an angle $\beta=90^{\circ}+2 A \omega(1-\omega) \phi$, where $2 \phi$ is the angle formed by the two twin variants in the tetragonal phase, with polarizations along [100] and 
[001], respectively, $\omega$ and $(1-\omega)$ are the volume fractions of the two variants, and A is a fitting parameter related to the volume fraction of domain walls or the size of the domains [95]. If neither an electric field nor uniaxial stress is applied, then the stress accommodation relations between the tetragonal and the parent cubic phase $\left(a_{c}\right)$ would also give: $\quad a_{a d}=a_{c}$. Adaptive phases of this sort have been previously observed in martensites [96]. The remarkable agreement between the above relationships and the experimental data reported for the monoclinic $\mathrm{M}_{C}$ phases in PMN$\mathrm{xPT}$ and PZN-xPT is illustrated in Refs. [94, 95]. According to this model the effect of an applied electric field would be to detwin the crystal, which would modify the relative fraction of domains with polarizations along [001] and [100] (changing $\omega$ ), effectively producing the rotation of the macroscopic polarization[95]. The adaptive model can explain characteristics of the monoclinic $\mathrm{M}_{C}$ phase previously considered as curiosities, such as the $b_{m}$ lattice parameter being a continuous extrapolation of the $a_{t}$ lattice parameter. Furthermore, these authors state that a similar approach to rhombohedral domains yields the monoclinic $\mathrm{M}_{A}$ phase observed in PZT and PZN-4.5PT. Experimental evidence of a high domain-wall density and domain structures at various length scales at the MPB of PMN-xPT observed by piezo-force microscopy has recently been reported by Bai et al. [97]. Moreover the nanotwin model of the monoclinic phase is also consistent with the large anisotropic broadening of the diffraction profiles around the MPB (with narrow 0k0 peaks) observed in PMN-xPT[29], as well as the phase coexistence often encountered over large temperature and compositional ranges [95].

Topolov and Ye $[66,98]$ consider a model of interpenetrating phases to explain the MPB of PMN-xPT. The phases are separated by planes with zero net strain. According to this model each sample consists of two phases and two different types of regions, each one containing both phases. In one type of region phase- 1 inclusions exist in a phase2 matrix, and vice versa for the second type of region. The elastic matching at the phase boundaries relates the lattice parameters of the intermediate monoclinic phase with those of the tetragonal and rhombohedral phases. The same elastic matching can be obtained in different ways; for example, with the coexistence of three phases in a more narrow composition region. This model could account for the two different phase diagrams reported for PMN-xPT $[29,33,34]$. In common with Viehland et al., this model explains the stability of the M phase at the MPB by stress accommodation at the interfaces of the $\mathrm{R}$ and $\mathrm{T}$ phases, as well as the relation between the lattice parameters of the monoclinic phase and those of the neighbouring phases. But, contrary to Viehland et al., it does not assume subnanometer domains of those phases but instead a true $\mathrm{M}_{C}$ phase in coexistence with at least one other phase. It is evident that more experimental work is needed in order to clarify these various pictures, and to find whether a single model applies both to PZT and to the relaxor single crystals.

\section{PIEZOELECTRIC RESPONSE}

In recent years much effort has been devoted towards the control of the performance of these technologicallyimportant materials [11, 99-102], but despite all the recent progress in understanding their fundamental behavior, the origin of the piezoelectric response in these materials is still a matter of debate and there is still some controversy about whether the piezoelectric properties of these materials are intrinsic or extrinsic in nature, if they are due to the ferroelectric domains or to the motion of the domain walls.

The adaptive theory described in the previous section suggests that the large electromechanical response in these materials around the MPB is due to detwinning, that is movement of domain walls[95]. A [001]-oriented electric field would increase the size of the tetragonal twins with polarization along the [001] direction, reducing those along the [100] direction, until they become visible to diffraction probes. During this process the diffraction probes will detect the rotation of the polarization within a $\mathrm{M}$ phase and a $\mathrm{M}$-to- $\mathrm{T}$ transformation. However, Damjanovic and coworkers[103] observed that, in PMN-33PT, the piezoelectric response is mainly intrinsic due to shear piezoelectricity, or polarization rotation, inside the domains, and that the multidomain state had a relatively small effect $(<20 \%)$ in the total response. This is again contrary to the analysis by Renault et al. for PZN-9PT [104], who propose that a large density of domain walls are present in these crystals, resulting in an important extrinsic component to the piezoelectric response. In agreement with the latter proposal are the experiements of Wada et al.[105] showing that the piezoelectric response of $\mathrm{KNbO}_{3}$ can be considerably improved by reducing the domain size. On the other hand, polarization switching observed in PZN-4.5PT by Davis et al. suggests that there is no domain growth but continuous renucleation instead [106]. For small opposing fields a quick polarization response occurs which is both reversible and non-dissipative (indicating no activation barrier), consistent with polarization rotation. At larger fields, nucleation rather than domain growth is observed. The broad distribution of times suggests nucleation across the crystal, probably at defect sites [107]. 
As discussed above, first-principles calculations point to polarization rotation of $\mathrm{R}$ crystals under an [001]-electric field between the $\mathrm{R}$ and $\mathrm{T}$ phase following several possible paths [20,61]. These calculations predict intermediate monoclinic phases, and even a triclinic phase, to be stabilized by the electric field during the rotation. These tetragonal, monoclinic and rhombohedral phases at the MPB are nearly degenerate[21], corresponding to flat energy surfaces, which explains why subtle differences in sample preparation or microstructure can lead to changes in phase stability. Phenomenological calculations are consistent with this picture[22]. In the light of these calculations Vanderbilt and Cohen suggested that the presence of monoclinic phases in ferroelectric perovskites arises as a consequence of unusually anharmonic free energies [22]. Highly anharmonic potentials have indeed been reported for lead perovskites[108]. The soft potentials at the MPB are responsible for the non-collateral anomalies in the dielectric and piezoelectric responses observed at the MPB, the so-called transverse instabilities[21, 109-116]. The presence of a ferro-ferro phase transition, such as that occurring at the MPB's or the tetragonal-orthorhombic transition in $\mathrm{BaTiO}_{3}$ with a change of polarization direction, is accompanied by a dielectric softening in the perpendicular direction[13, 62], associated with a shear component of the piezoelectric tensor. An extensive review by D. Damjanovic on this topic has been published during the writing of this manuscript[117].

From first-principles calculations on a tetragonal PZT crystal with random Zr/Ti distribution, Bellaiche et al. [21] have shown that in the proximity of the T-M boundary the shear piezoelectric coefficient $d_{15}$, which is related to the rotation of the polarization from [001] to [111], is greatly enhanced, thus explaining the observed piezoelectric response. More recently, Wu and Krakauer were able to calculate the electromechanical response in an ordered monoclinic phase and obtain very large piezoelectric coefficients, similar to the experimental ones[111]. This explicitly shows that polarization rotation alone can explain the observed response at the MPB. As discussed by these authors, although this mechanism could be generalized to other ferroelectrics such as $\mathrm{BaTiO}_{3}$ (keeping in mind that, as pointed out in Ref. [40], the symmetry of a rhombohedral crystal under a [001] field, or a tetragonal crystal under a [111]field, is monoclinic in general), it is only at the MPB where the energies of the different phases are close enough, or the energy surfaces flat enough, for the symmetry of the crystal to change under the available E-field. Hydrostatic pressure, as discussed above[57], or uniaxial stress along the polar direction could help to bring the free energies closer to each other.[116]

Very recently, surprisingly high $d_{33}$ values of $2000 \mathrm{pC} / \mathrm{N}$ have been obtained by Chu et al. [118] in $\mathrm{BaTiO}_{3}$ poled along the [720] direction. This direction is perpendicular to a cleavage plane and allows high values of the electric field to be applied without fracturing the crystal. This leads to a very large deformation and maximum strain values of $0.6 \%$ with fields below $10 \mathrm{kV} / \mathrm{cm}$. However, with slight deviations from this poling direction, the $d_{33}$ value is reduced by one-half. Further theoretical and experimental work is needed to understand this strikingly high values in the piezoelectric response of $\mathrm{BaTiO}_{3}$.

From the above discussion, it is clear that, despite the many unknowns, our understanding of the piezoelectric response in perovskites has improved considerably in the last couple of years. Accordingly, although it is not yet clear that a monoclinic unit cell is a necessary condition for large piezoelectric responses, it seems nevertheless wise to focus the search for highly responsive dielectric and piezoelectric systems around low symmetry phases that will allow polarization rotation, namely, those polar phases that, independent of the local symmetry, appear to be monoclinic or triclinic from x-ray or neutron diffraction measurements. These phases will be likely to appear in regions bridging higher symmetry phases of similar energy.

\section{ACKNOWLEDGEMENTS}

It is with much sadness that we dedicate this article to the memory of Gen Shirane. We had the great privilege of working closely with Gen on structural investigations of the morphotropic phase boundaries in different lead oxide systems during his last five years at Brookhaven National Laboratory, and we have profited enormously from his deep insight into the physics of the problem, and his unerring instinct for doing the right experiment. Working with him was an entertaining, rewarding and unforgettable experience!

We would like to thank Ilya Grinberg, Andrew Rappe and Yu Wang for sharing with us their unpublished results, Ben Burton for an interesting conversation about the importance of the order of the tetragonal-monoclinic phase transition, and Mike Glazer for many illuminating discussions. Financial support from the U. S. Department of Energy, Division of Materials Sciences, under Contract No. DE-AC02-98CH10886, is also acknowledged. 
[1] G. Shirane and A. Takeda, J. Phys. Soc. Jpn. 7, 5 (1952).

[2] G. Shirane, K. Suzuki, and A. Takeda, J. Phys. Soc. 7, 12 (1952).

[3] G. Shirane and K. Suzuki, J. Phys. Soc. Jpn. 7333 (1952).

[4] E. Sawaguchi, J. Phys. Soc. Jpn. 8, 615 (1953).

[5] B. Jaffe, W.R. Cook, and H. Jaffe, Piezoelectric Ceramics (Academic Press, London, 1971).

[6] V.A. Isupov, Solid State Commun. 17, 1331 (1975).

[7] K. Kakegawa, J. Mohri, T. Takahashi, H. Yamamura, and S. Shirasaki, Solid State Commun. 24, 769 (1977).

[8] S.K. Mishra, D. Pandey, and A.P. Singh, Appl. Phys. Lett. 69, 1707 (1996).

[9] W. Cao, and L.E. Cross, Phys Rev B 47, 4825 (1993).

[10] A. M. Glazer, P. A. Thomas, K. Z. Baba-Kishi, G. K.H. Pang, and C.W. Tai, Phys. Rev. B 70, 184123 (2004).

[11] S-E. Park, and T. R. Shrout, J. of Appl. Phys. 82, 1804 (1997).

[12] M.K. Durbin, J.C. Hicks, S-E. Park, and T.R. Shrout, J. Appl. Phys. 87, 8159 (2000).

[13] X-H. Du, J. Zheng, U. Belegundu, and K. Uchino, Appl. Phys. Lett. 72, 2421 (1998).

[14] D.V. Taylor, and D. Damjanovic, Appl. Phys. Lett. 76, 1625 (2000).

[15] R. Guo, L. E. Cross, S-E. Park, B. Noheda, D.E. Cox, and G. Shirane, Phys. Rev. Lett. 84, 5423 (2000).

[16] J-T. Reszat, A.E. Glazonounov, and M.J. Hoffmann, J. European Ceram. Soc. 21, 1349 (2001).

[17] B. Noheda, D. E. Cox, G. Shirane, J. A. Gonzalo, L. E. Cross, and S-E. Park, Appl. Phys. Lett. 74, 2059 (1999).

[18] B. Noheda, D. E. Cox, G. Shirane, R. Guo, B. Jones, and L.E. Cross, Phys. Rev. B 63014103 (2000).

[19] B. Noheda, J. A. Gonzalo, L. E. Cross, R. Guo, S-E. Park, D.E. Cox, and G. Shirane, Phys. Rev. B 61, 8687 (2000).

[20] H. Fu and R. E. Cohen, Nature 403, 281 (2000).

[21] L. Bellaiche, A. Garcia, and D. Vanderbilt, Phys. Rev. Lett. 84, 5427 (2000).

[22] D. Vanderbilt and M. H. Cohen, Phys. Rev. B 63, 094108 (2001),

[23] A. K. Singh and D. Pandey, J. Phys.: Condens. Matter 13, L931 (2001).

[24] G. Xu, H. Luo, H. Xu, and Z. Yin, Phys Rev B 64, 020102 (2001).

[25] D.E. Cox, B. Noheda, G. Shirane, Y. Uesu, K. Fujishiro, and Y. Yamada, Appl. Phys. Lett. 79, 400 (2001).

[26] Z-G. Ye, B. Noheda, M. Dong, D.E. Cox, and G. Shirane, Phys. Rev. B 64, 184114 (2001).

[27] Y. Yamada, Y. Uesu, M. Matsuda, K. Fujishiro, D. E. Cox, B. Noheda, and G. Shirane, J. Phys. Soc. Jpn 71, 966 (2002).

[28] J-M. Kiat, Y. Uesu, B. Dkhil, M. Matsuda, C. Malibert and G. Calvarin, Phys Rev B 65, 064106 (2002).

[29] B. Noheda, D. E. Cox, G. Shirane, J. Gao, and Z.-G. Ye, Phys. Rev. B 66, 054104 (2002).

[30] D. La-Orauttapong, B. Noheda, Z-G. Ye, P. M. Gehring, J. Toulouse, D.E. Cox, and G. Shirane, Phys Rev B 65, 144101 (2002).

[31] Y. Uesu, M. Matsuda, Y. Yamada, K. Fujishiro, D.E. Cox, B. Noheda, and G. Shirane, J. Phys. Soc. Jpn 71, 960 (2002).

[32] B. Dkhil, J.-M. Kiat, G. Calvarin, G. Baldinozzi, S. B. Vakhrushev, and E. Suard, Phys. Rev. B 65, 024204 (2002).

[33] A. K. Singh and D. Pandey, Phys. Rev. B 67, 064102 (2003); A. K. Singh, D. Pandey, and O. Zakharko, Phys. Rev. B 68, $172103(2003)$.

[34] D. Zekria, V. A. Shuvaeva, and A. M. Glazer, J. Phys.: Condens. Matter 17, 1593 (2005).

[35] A-E. Renault, H. Dammak, G. Calvarin and P. Gaucher, J. Appl. Phys. 97, 044105 (2005)

[36] R. Haumont, B. Dkhil, J. M. Kiat, A. Al-Barakaty, H. Dammak, and L. Bellaiche, Phys. Rev. B. 68, 014114 (2003).

[37] R. Haumont, A. Al-Barakaty, B. Dkhil, J. M. Kiat, and L. Bellaiche, Phys. Rev. B 71, 104106 (2005).

[38] B. Noheda, Current Opinion in Solid State and Materials Science 6, 27 (2002).

[39] A. Amin and D. Viehland, J. Appl. Phys. 96, 5140 (2004).

[40] E. H. Kisi, R. O. Piltz, J. S. Forrester, and C. J. Howard, J. Phys.: Condens. Matter 15, 3631 (2003).

[41] M. Fornari and D.J. Singh, Phys Rev B 63, 092101 (2001).

[42] Ragini, S. K. Mishra, D. Pandey, H. Lemmens, and G. Van Tendeloo, Phys. Rev. B 64, 054101 (2001).

[43] R. Ranjan, Ragini, S.K. Mishra, D. Pandey, and B. J. Kennedy, Phys. Rev. B 65, 060102 (2002).

[44] D. M. Hatch, H. T. Stokes, R. Ranjan, Ragini, S.K. Mishra, and D. Pandey, Phys. Rev. B 65212101 (2002).

[45] J. Frantti, S. Ivanov, S. Eriksson, H. Rundolf, V. Lantto, J. Lappalainen, and M. Kakihana, Phys. Rev. B 66, 064108 (2002).

[46] B. Noheda, L. Wu, and Y. Zhu, Phys. Rev. B. 66, 060103 (2002).

[47] D.E. Cox, B. Noheda, and G. Shirane, Phys. Rev. B. 71, 134110 (2005).

[48] R. Ranjan, A.K. Singh, Ragini, and D. Pandey, Phys. Rev. B 71, 134110 (2005).

[49] J. Frantti, arXiv:cond-mat/0504432 v1 18 April 2005.

[50] J. Frantti, S. Eriksson, S. Hull, V. Lantto, H. Rundolf, and M. Kakihana, J. Phys.: Condens Matter 15, 6031 (2003).

[51] In conventional Rietveld analysis, the peak profile model is assumed to have a smooth dependence on $2 \theta$, whereas in practice it is often found that there is additional broadening associated with different $h k l$ classes of reflections due to microstructural effects such as size, strain and local order.

[52] D. I. Woodward, J. Knudsen, and I. M. Reaney, Phys. Rev. B 72, 104110 (2005)

[53] A. M. Glazer, Acta Cryst. B28, 3384 (1972)

[54] A. Sani, B. Noheda, I. A. Kornev, L. Bellaiche, P. Bouvier, and J. Kreisel, Phys. Rev. B 69, 020105 (2004).

[55] J. Rouquette, J. Haines, V. Bornand, M. Pintard, Ph. Papet, C. Bousquet, L. Konczewicz, F. A. Gorelli, and S. Hull, Phys. Rev. B 70, 014108 (2004). 
[56] J. Rouquette, J. Haines, V. Bornand, M. Pintard, Ph. Papet, W. G. Marshall, and S. Hull Phys. Rev. B 71, 024112 (2005).

[57] Z. G. Wu and R. E. Cohen, Phys. Rev.Lett. 95, 037601 (2005).

[58] B. Noheda, D. E. Cox, G. Shirane, S.-E. Park, L. E. Cross, and Z. Zhong, Phys. Rev. Lett. 86, 3891 (2001)

[59] B. Noheda, Z. Zhong, D. E. Cox, G. Shirane, S-E. Park, and P. Rehrig, Phys. Rev. B 65, 224101 (2002)

[60] H. Cao, F.M. Bai, J.F. Li, D. Viehland, G.Y. Xu, H. Hiraka and G. Shirane, J. of Appl. Phys. 97, 094101 (2005)

[61] L. Bellaiche, A. Garcia, and D. Vanderbilt, Phys Rev B 64, 060103 (2001)

[62] D. Viehland, A. Amin, and J. F. Li, Appl. Phys. Lett. 79, 1006 (2001)

[63] H. Cao, F.M. Bai, N.G. Wang, J.F. Li, D. Viehland, G.Y. Xu and G. Shirane, Phys. Rev. B 72, 064104 (2005)

[64] A-E. Renault, H. Dammak, G. Calvarin, M. Pham Thi, and P. Gaucher, Jpn. J. Appl. Phys. 41, 3846 (2002).

[65] V. Y. Topolov and A.V. Turik, J. Phys.: Condens. Matter 13, 771 (2001).

[66] V. Y. Topolov, J. Phys.: Condens. Matter 16, 2455 (2004).

[67] J.Frantti, S. Eriksson, S. Hull, S. Ivanov, V. Lantto, J. Lappalainen, and M. Kakihana, J. European Ceram. Soc. 241141 (2004).

[68] K. C. V. Lima, A. G. Souza Filho, A. P. Ayala, J. Mendes Filho, P. T. C. Freire, F. E. A. Melo, E. B. Araujo, and J. A. Eiras, Phys. Rev. B 63, 184105 (2001).

[69] C. A. Guarany, L. H. Z. Pelaio, E. B. Araujo, K Yukimitu , J C S Moraes, and J A Eiras, J. Phys.: Condens. Matter 15, 4851 (2003).

[70] R.-A. Eichel , K.-P. Dinse, H. Kungl, M.J. Hoffmann, A. Ozarowski, J. van Tol, and L.C. Brunel, Appl. Phys. A: Materials Science and Processing 80, 51 (2005).

[71] Ragini, Rajeev Ranjan, S. K. Mishra, and Dhananjai Pandey, J. Appl. Phys. 92, 3266 (2002).

[72] B. Noheda, J. A. Gonzalo, A. C. Caballero, C. Moure, D. E. Cox, and G. Shirane, Ferrolectrics 237, 237 (2000).

[73] B. Noheda, D.E. Cox, and G. Shirane, Ferroelectrics 267,147 (2002).

[74] R. Bertram, G. Reck, and R. Uecker, J. Cryst. Growth 253, 212 (2003).

[75] Xu et al. Phys. Rev. B. 67, 104102 (2003)

[76] Xu et al., Phys. Rev. B 68, 212410 (2003)

[77] Gehring et al., J. Phys.: Condens. Matt 16, 7113 (2004)

[78] Xu et al., Appl. Phy. Lett. 84, 3975 (2004)

[79] Colon et al., Phys. Rev. B 70, 172204 (2004)

[80] Ohwada et al. J. Phys. Soc. Japan 702778 (2001)

[81] A. J. Bell and E. Furman, Ferroelectrics 293, 19 (2003).

[82] C. Malibert, B. Dkhil, J. M. Kiat, D. Durand, J. F. Berar, and A. Spasojevic-de-Bire, J. Phys.: Condens. Matter 9, 7485 (1997).

[83] A. M. Glazer, S. A. Mabud, and R. Clarke, Acta Cryst. B 34, 1060 (1978).

[84] S. Teslic, T. Egami, and D. Viehland, J. Phys. Chem. Solids 57, 1537 (1996).

[85] D. L. Corker, A. M. Glazer, R. W. Whatmore, A. Stallard, and F. A. Fauth, J Phys.: Condens. Matter 10, 6251 (1998).

[86] W. Dmowski, T. Egami, L. Farber, and P.K. Davies, AIP Conf. Proc., 582, 33 (2001).

[87] I. Grinberg, V. R. Cooper, and A. M. Rappe, Nature 419, 909 (2002); Phys. Rev. B 69, 144118 (2004)

[88] I. Grinberg and A. M. Rappe, Phys. Rev. B 70, 220101 (2004)

[89] I. Grinberg, M. R. Suchomel, P. K. Davies and A. M. Rappe, J. Appl. Phys. (in press); arXiv:cond-mat/0509424

[90] Y-H. Shin, V. R. Cooper, I. Grinberg, and A. M. Rappe, Phys. Rev. B 71, 054104 (2005)

[91] I. Grinberg, Y-H. Shin, and A. M. Rappe (private communication)

[92] D. Cao, I.-K. Jeong, R. H. Heffner, T. Darling, J.-K. Lee, F. Bridges, J.-S. Park, and K.-S. Hong, Phys. Rev. B 70, $224102(2004)$.

[93] D. Viehland, J. Appl. Phys. 88, 4794 (2000).

[94] Y. M. Jin, Y. U. Wang, A. G. Khachaturyan, L. F. Li, and D. Viehland, Phys. Rev. Lett. 91, 197601 (2003) ; Y. Jin, Y. Wang, A. G. Khachaturyan, J. F. Li, and D. Viehland, J. Appl. Phys. 94, 3629 (2003).

[95] Y.U. Wang, Phys. Rev. B. (submitted)

[96] S. M. Shapiro, B. X. Yang, G. Shirane, Y. Noda, and L. E. Tanner, Phys. Rev. Lett. 62, 161 (1989).

[97] F. Bai, J. Li, and D. Viehland, J. Appl. Phys. 97, 054103 (2005).

[98] V. Y. Topolov and Z.-G. Ye, Phys. Rev. B 70, 094113 (2004).

[99] D. Viehland, J. F. K. Li, J. Appl. Phys. 90, 2995 (2001)

[100] M. Ozgul, S. Trolier-McKinstry, and S. A. Randall, J. Appl. Phys. 95, 4296 (2004).

[101] S. Trolier McKinstry and P. Muralt, J. Electroceramics 12, 7 (2004).

[102] J. Y. Li, R. C. Rogan, E. Ustundag, and K. Bhattacharya, Nature Materials 4, 776 (2005)

[103] D. Damjanovic, M. Budimir, M. Davis, and N. Setter, Appl. Phys. Lett. 83, 527 (2003).

[104] A-E. Renault, H. Dammak, G. Calvarin, M. Pham Thi, and P. Gaucher, Jpn. J. Appl. Phys. 42, 6477 (2003).

[105] S. Wada et al., Jpn. J. of Appl. Phys. 43, 6692 (2004)

[106] M. Davis, D. Damjanovic, and N. Setter, J. Appl . Phys. 97, 064101 (2005).

[107] C. Jullian, J. F. Li, and D. Viehland, J. Appl. Phys. 95, 5671 (2004).

[108] J-M. Kiat. G. Baldinozzi, M. Dunlop, C. Malibert, B. Dkhil, C. Menoret, O. Masson, M-T. Fernandez-Diaz, J. Phys.:Condens. Matter 12, 8411 (2000).

[109] Y. Ishibashi and M. Iwata, Jpn. J. Appl. Phys. 37, L985 (1998).

[110] M. Iwata and Y. Ishibashi, Topics in Applied Physics. 98, 127 (2005). 
[111] Z. Wu and H. Krakauer, Phys. Rev. B 68, 014112 (2003).

[112] C. Bungaro and K. Rabe, Phys. Rev. B. 69, 184101 (2004).

[113] M. Budimir, D. Damjanovic, and N. Setter, J. Appl. Phys. 94, 6753 (2003).

[114] D. Damjanovic, F. Brem, and N. Setter, Appl. Phys. Lett. 80, 652 (2002).

[115] M. Budimir, D. Damjanovic, and N. Setter, Appl. Phys. Lett. 85, 2890 (2004).

[116] M. Budimir, D. Damjanovic, and N. Setter, Phys. Rev. B 72, 064107 (2005).

[117] D. Damjanovic, J. Am. Ceram. Soc. 88, 2663 (2005)

[118] R. Chu, Z. Xu, G. Li, H. Zeng, H. Yu, H. Luo, and Q. Yin, Appl. Phys. Lett. 86, 012905 (2005). 